\title{
El arte como instrumento para el desarrollo de la empatía
}

\section{Art as an instrument to develop empathy}

En un artículo anterior (El arte como instrumento educativo en medicina) hemos expuesto la idea de introducir el arte como recurso docente en la formación de los profesionales de la salud, propuesta basada en la capacidad que el lenguaje simbólico del arte tiene de reflejar la dimensión emocional humana. ${ }^{1}$

En el presente artículo analizaremos uno de los principales componentes del mundo de lo inefable: la actitud empática. La empatía constituye la sublime capacidad humana de vivir "en carne propia" los sentimientos de otra persona, y de poder incluso hacerlo evidente en la relación con ella, ya sean estos sentimientos agradables (alegría, alivio, etc.) o desagradables (tristeza, dolor, etc.). ${ }^{2,3}$ Cuando lo que se experimenta es el sufrimiento del otro, la empatía toma el nombre de compasión.

Por otra parte, no debe confundirse la compasión con la piedad: si bien en ambas se logra experimentar en algún grado el sufrimiento del otro, en la compasión se lo hace desde un plano emocional de igualdad, mientras que en la piedad se lo hace desde un plano de supuesta superioridad. Asimismo, a la empatía se la debe distinguir de la simpatía, en la cual a diferencia de lo que ocurre en la empatía, se produce un contagio emocional, de modo que quien experimenta simpatía no sabe bien cuáles son sus emociones y cuáles las del otro, perdiendo así objetividad. Debe distinguirse también a la empatía del mero trato amable (la cordialidad) y de la simple pena inspirada por el infortunio ajeno (la lástima), pues en ambas situaciones no se comparten las emociones del otro. ${ }^{4}$

El ejercicio de la empatía requiere de la conjunción de una serie de condiciones: por un lado el esfuerzo y la apertura para reconocer al otro como portador de emociones, y por otro, el coraje y la paciencia para experimentar transitoriamente dichas emociones en uno mismo, a fin de comprenderlas, pues su fin último es entender lo que el otro experimenta. Por esta razón, es que la empatía exige, tras haber logrado una comunión afectiva, el "distanciamiento" respecto del otro, a fin de evaluar objetivamente la situación y poder decidir y actuar adecuadamente. De ahí que para que la experiencia empática se traduzca en ayuda efectiva, ésta debe ser de carácter transitorio. ${ }^{2-4}$

Resulta entonces que, si bien todos los seres humanos poseen la capacidad innata de sentir empatía, ésta puede optimizarse, a través de su ejercitación y mayor comprensión por medio de la lectura (literatura), contemplación (pintura, escultura, danza, cine) y/o escucha (música) de obras maestras del arte universal; pues la genialidad de sus autores les ha permitido infundirles una fuerte eficacia simbólica, a tal punto que el contacto con este tipo de material resulta transformador, pues torna a quien impregna en una persona más sensible y empática. ${ }^{5-10}$

A continuación veamos algunos ejemplos de la literatura universal:

En "Ulysses" de James Joyce, existen diversos pasajes que muestran la gran capacidad empática de su personaje principal (Leopold Bloom); como cuando Bloom se acuerda de la señora Purefoy, quien acaba de dar a luz, y al hacerlo sufre él mismo las sensaciones del trabajo de parto:

¡Pobre señora Purefoy! ¡Imagínese, tres días quejándose en la cama! (...). La cabeza del chico demasiado grande: fórceps.

Durante la experiencia empática, la situación vivida por el otro encuentra eco en el mundo interior de quien lo observa y esa conexión se hace posible gracias a ese "trasbordador al pasado" que es la memoria sentimental. Es que solo se puede sentir lo que se reconoce y si se lo identifica es porque se lo ha padecido. Este tipo de memoria es descripta magistralmente por Marcel Proust en "Por el camino de Swann":

...Pero en el mismo instante en que aquel trago con las migas del bollo, tocó mi paladar, me estremecí, fija mi atención en algo extraordinario que ocurría en mi interior. Un placer delicioso me invadió, me aisló, sin noción de lo que lo causaba. Y él me convirtió las vicisitudes de la vida en indiferentes, sus desastres en inofensivos y su brevedad en ilusoria, todo del mismo modo que opera el amor... ¿De dónde podría venirme aquella alegría tan fuerte? Me daba cuenta de que iba unida al sabor del té y del bollo, pero le excedía en mucho y no debía de ser de la misma naturaleza...

En su obra "La Montaña Mágica", Thomas Mann describe esa sensación de abolición de las coordenadas de tiempo y espacio que envuelve a quien atraviesa una experiencia empática:

“...De pronto se encontró transportado a aquel estado primigenio del alma de cuyo arquetipo había surgido un sueño que había tenido algunas noches atrás... Y fue una sensación tan fuerte, tan infinita, se vio tan enteramente transportado a aquel momento y a aquel lugar del pasado, hasta el punto de perder la conciencia del tiempo y del espacio, que se hubiera dicho 
que era un cuerpo inanimado el que yacía en el banco, mientras que el verdadero Hans Castorp se hallaba muy lejos, en un tiempo y un espacio remotos..."

La empatía beneficia a quien la recibe y a quien la ejerce, al hacer que el primero sea mejor comprendido y el segundo más comprensivo respecto de la situación experimentada por el otro. Asimismo, quien ejerce la empatía, vive en definitiva "muchas vidas" y va adquiriendo paulatinamente una extensa experiencia de vida y sabiduría, mucha más de la que jamás podría haber logrado desde los estrechos límites de su ego. ${ }^{2,3}$

Concluimos entonces en lo valioso que resulta la incorporación del arte como un instrumento educativo en pos de lograr un mayor desarrollo de la capacidad empática en los profesionales de la salud.

$$
\begin{array}{r}
\text { Dr. Carlos G. Musso y Dra. Paula A. Enz } \\
\text { Escuela de Medicina, } \\
\text { Instituto Universitario del } \\
\text { Hospital Italiano de Buenos Aires, } \\
\text { Argentina }
\end{array}
$$

http:/ /dx.doi.org/10.5546/aap.2015.101

\section{REFERENCIAS}

1. Musso CG, Enz PA. El arte como instrumento educativo en medicina. Arch Argent Pediatr 2014;112(6):494-5.

2. Musso CG. La empatía en la mitología. Primera parte. Medicina \& Cultura [Internet] 2012 [Acceso: 29 de diciembre de 2014];6(61). Disponible en: http:/ / www.medicinaycultura.org/61/Articulo_04.htm

3. Musso CG. La empatía en la mitología. Segunda parte. Medicina \& Cultura [Internet] 2012 [Acceso: 29 de diciembre de 2014];6(62). Disponible en: http:/ / www.medicinaycultura.org/62/Articulo_01.htm

4. Musso CG, Enz PA. Arte y naturaleza humana XII. Rev Hosp Ital B Aires 2013;33(2):71-2.

5. Job AJ. Comunicación y sentido. Fundamentos del respeto. Buenos Aires: De los cuatro vientos; 2011.

6. Musso CG, Enz P. La empatía en la obra de James Joyce. Primera parte: Ulysses. Medicina \& Cultura [Internet] 2012 [Acceso: 29 de diciembre de 2014];6(69). Disponible en: http://www.medicinaycultura.org/69/Articulo_07.htm

7. Musso CG, Enz P. La empatía en la obra de James Joyce. Segunda parte: Finnegans Wake. Medicina \&Cultura[Internet] 2012 [Acceso: 29 de diciembre de 2014]; 6(70). Disponible en: http://www.medicinaycultura.org/70/Articulo_05. htm

8. Musso CG, Enz P. La empatía en la obra de Thomas Mann. Medicina \& Cultura [Internet] 2013 [Acceso: 29 de diciembre de 2014];7(78). Disponible en: http:/ / www.medicinaycultura.org/78/Articulo_04.htm

9. Musso CG, Enz P. La empatía y la memoria proustiana. Medicina \& Cultura [Internet] 2013 [Acceso: 29 de diciembre de 2014];7(79). Disponibleen:http:/ / www.medicinaycultura.org/79/Articulo_01.htm

10. Haber A. Jung y el principio de sincronicidad: arquetipos y símbolos. Buenos Aires: Rueda; 1956.

\title{
Debilidades de la prescripción electrónica en pacientes pediátricos hospitalizados
}

\author{
Weaknesses of assisted electronic prescription in hospitalized pediatric inpatients
}

Los errores de medicación (EM) constituyen un problema sanitario que, en la población infantil, adquiere especial relevancia. Como es sabido, el riesgo de sufrir efectos adversos derivados de errores de medicación en esta población es mayor y, además, su trascendencia es más notable, ya que pequeños errores que, en adultos serían tolerados, en niños, pueden ocasionar daños significativos.

El análisis y la identificación de las causas que desencadenan EM son fundamentales para adoptar estrategias y medidas correctoras con el fin de reducir riesgos para futuros pacientes. Hay que tener en cuenta que, en niños, existen factores propios que contribuyen a que los EM sean diferentes a los de adultos y que sus causas sean específicas. El conocimiento de estas diferencias es importante a la hora de adecuar a la población infantil las estrategias para su prevención de errores.

En este ámbito, las nuevas tecnologías, al igual que en pacientes adultos, están teniendo un papel fundamental en la reducción de riesgos. Permiten la detección de errores y la puesta en marcha de medidas que logran reducirlos en una población especialmente vulnerable. En la actualidad, dado que los EM ocurren, en su mayoría, en la fase de prescripción, una de las herramientas más empleadas para disminuirlos es el sistema de prescripción electrónica asistida (PEA). Estos programas incluyen, además, plataformas de ayuda, que facilitan la toma de decisiones. ${ }^{1}$

En cuanto a los artículos publicados sobre el efecto de la PEA en los EM, estos son más 
escasos en la población pediátrica que en adultos y muestran tasas dispares de reducción de errores. Además, sus resultados son difícilmente comparables debido a la alta variabilidad y la falta de consenso en la definición de EM, efectos adversos, sus métodos de detección y programas de prescripción empleados. ${ }^{2}$ No obstante, el hecho de que, en su mayoría, estos trabajos hayan demostrado su eficacia, ha llevado a organismos, como la American Academy of Pediatrics, a considerarla la principal estrategia para reducir los errores en la prescripción e incrementar la seguridad de los niños hospitalizados y en urgencias. En la bibliografía, hay recogidas también recomendaciones sobre puntos clave que estos sistemas deberían proporcionar para adaptarse a la prescripción en pediatría. ${ }^{3}$

Sin embargo, a pesar de las ventajas demostradas por la PEA, presenta algunos puntos débiles. Así, algunos autores han demostrado que este sistema induce nuevos errores de medicación y, de hecho, la mayoría están relacionados con su uso.

En un trabajo recientemente publicado, ${ }^{4}$ se observó que, en pacientes adultos, la incidencia de errores de prescripción se redujo con la PEA al $0,8 \%$ y que la mayoría de ellos $(77,7 \%)$ se asociaron con el empleo de esta tecnología.

Uno de los fallos más frecuentes fue la selección incorrecta del medicamento en una lista alfabética; este hecho puede ocasionar un daño grave, dado que, en ellas, no existe relación entre un fármaco y el siguiente en cuanto a su acción terapéutica. En niños, también se han notificado errores por esta causa. ${ }^{5}$ Este tipo de fallo se podría subsanar si la selección de medicamentos se hiciera sobre listas de medicamentos agrupados por indicación terapéutica o asociadas a ciertas patologías.

Otro tipo de error prevalente derivó de la falta de conocimiento del programa, que hizo que el prescriptor registrara datos en un lugar inadecuado o recurriera al uso de campos destinados a texto libre, lo cual generó también numerosos errores. Algunos autores han comprobado que el riesgo de error al utilizar texto libre en la prescripción es cinco veces mayor que si se estandarizan textos estructurados. ${ }^{3}$ Así, por ejemplo, se han detectado, por este motivo, fallos (como duplicidades) debidos a la prescripción de fármacos tanto en la lista electrónica como en texto libre o que no se desencadenaran alertas por registro inadecuado de alergias. ${ }^{4,5}$ Este registro inadecuado de datos en el programa puede tener consecuencias para el paciente potencialmente graves. Por otro lado, las alertas que proporciona la PEA han demostrado su utilidad; sin embargo, su sobrecarga puede llevar a que sean ignoradas por el clínico.
Además de los nuevos fallos inducidos por la PEA, en la población pediátrica, otro punto débil importante de este método de prescripción es la dificultad añadida de adaptar los programas diseñados para adultos. Su adecuación, en muchos casos, es difícil y, en ocasiones, inefectiva y puede generar nuevos errores. Habitualmente, muchos hospitales se enfrentan a este problema, ya que atienden a ambos tipos de población. Esta falta de adecuación complica, en gran medida, su uso por parte de los pediatras y requiere un alto grado de especialización y destreza en su manejo. El principal inconveniente que se les plantea es que los soportes de ayuda a la prescripción, herramienta que ha demostrado mayor eficacia en la reducción de errores, están basados en indicaciones de tratamiento para población adulta, lo que los hace inoperativos para su prescripción en niños. Así, por ejemplo, la propuesta de dosis habitual proporcionada por el programa para pacientes adultos queda anulada en niños, puesto que depende de la edad y el peso, y además, estos sistemas, en muchos casos, tampoco facilitan el acceso a bases de datos de información de medicamentos en pediatría.

Otro problema que puede contribuir a fallos en la dosificación es la omisión del peso, dato imprescindible en la prescripción pediátrica para que se desencadenen alertas de dosis máximas o mínimas. En este sentido, la exigencia de cumplimentación obligatoria de este campo antes de iniciar la prescripción de fármacos sería otro aspecto que reduciría este tipo de errores.

Un inconveniente también específico de la farmacoterapia en niños, a diferencia de la prescripción en adultos, es la falta de disponibilidad de presentaciones de medicamentos adecuadas en concentración y forma farmacéutica a la población infantil. Este hecho obliga a su manipulación y a la elaboración de fórmulas magistrales por parte del Servicio de Farmacia y su inclusión en la base de datos para que estén disponibles para la prescripción. A menudo, en estos programas, es difícil la localización de estas fórmulas para el prescriptor, que puede acabar recurriendo a la selección inadecuada de presentaciones comercializadas, lo cual podría desencadenar nuevos errores. Además, en pediatría, existe el hábito de prescribir la dosis por volumen, de tal modo que, si variara la concentración de la presentación de un medicamento o de una formulación, al mantener el volumen prescrito, se modificaría la dosis a administrar y se podría producir un error de infra- o sobredosificación.

Como conclusión, diremos que, a pesar de que el sistema de PEA en población infantil ha 
demostrado eficacia en la reducción de errores de medicación, consideramos que actualmente presenta dos importantes puntos débiles: por un lado, la aparición de nuevos errores asociados al uso de estos programas y, por otro, la complejidad para adecuarlos a la prescripción de tratamientos en niños. La estandarización de estas aplicaciones informáticas para su utilización en población infantil, así como la disponibilidad de enlaces que proporcionen información actualizada sobre indicaciones y condiciones de uso en pediatría, son necesarias para mejorar la seguridad de los pacientes pediátricos.

Asimismo, para minimizar riesgos, es imprescindible que se tenga un adecuado conocimiento de estos programas por parte de los usuarios, lo cual exige una continua y específica formación del personal sanitario encargado del cuidado de los niños hospitalizados.

Dra. Margarita Ruano Encinar Dra. Elena Villamañán Bueno Servicio de Farmacia
Prof. Dr. Rodolfo Álvarez-Sala Walther

Servicio de Neumología

Hospital Universitario La Paz. Madrid. margarita.ruano@salud.madrid.org

http:/ /dx.doi.org/10.5546/aap.2015.102

\section{REFERENCIAS}

1. Conroy S, Sweis D, Planner C, Yeung V, et al. Interventions to reduce dosing errors in children. A systematic review of the literature. Drug Saf 2007;30(12):1111-25.

2. Wong ICK, Ghaleb MA, Franklin BD, Barber N. Incidence and nature of dosing errors in paediatric medications. A systematic review. Drug Saf 2004;27(9):661-70.

3. Maat B, Au YS, Bollen CW, van Vaught AJ, et al. Clinical pharmacy interventions in paediatric electronic prescriptions. Arch Dis Child 2013;98(3):222-7.

4. VillamañánE,Larrubia Y,RuanoM, VélezM, etal.Potential medication errors associated with computer prescriber order entry. Int J Clin Pharm 2013;35(4):577-83.

5. Walsh KE, Landrigan CP, Adams WG, Vinci RJ, et al. Effect of computer order entry on prevention of serious medication errors in hospitalized children. Pediatrics 2008;121(3):e421-7.

\section{¿Cómo escribir el resumen de una publicación científica?}

\section{Writing a scientific paper abstract}

Las reuniones científicas tienen entre sus objetivos principales intercambiar experiencias entre los participantes. La forma de hacer conocer estas experiencias suele ser la de un "Resumen", entendiendo por tal el resumen de una investigación original, presentación de caso clínico o experiencia profesional. Se trata de una pieza de redacción científica que, aunque refiera a otra de mayor extensión, debe ser absolutamente autónoma para permitir la comprensión del conocimiento que se pretende transmitir.

La capacidad de transmitir en muy pocas palabras (usualmente menos de 300) la idea que queremos hacer llegar a nuestros colegas es una habilidad que, como otras en nuestra profesión, puede aprenderse. Por otra parte, esta limitación de espacio nos obligará a condensar nuestro trabajo seleccionando solo lo mejor de él. ${ }^{1}$

En el caso de las investigaciones, es práctica generalizada que el Resumen sea "estructurado", es decir, que cuente con secciones fijas preestablecidas. ${ }^{2}$ Con ligeras modificaciones, se acepta que incluya "Introducción", "Métodos", "Resultados" y "Conclusiones".

La "Introducción" debe contener al menos una frase sobre la justificación del estudio (por qué se debía realizar la investigación) y establecer claramente el objetivo de la investigación (la pregunta de investigación). Los "Métodos" deben incluir el mínimo de elementos que permita juzgar la adecuación del estudio; esto comprende el diseño, la población (principal criterio de inclusión), la forma de recolección de datos, la medida de resultado principal, la principal forma de análisis, el lugar y momento donde se realizó el estudio. La sección de "Resultados" debe incluir solo los principales, incluyendo consideraciones descriptivas básicas seleccionadas (población, frecuencias) y la valoración de la variable de resultado primario; los resultados secundarios podrán incluirse en función de su trascendencia y del espacio disponible. La "Conclusión" incluirá la respuesta a la pregunta de investigación y, eventualmente, alguna recomendación en base a los resultados obtenidos o alguna consideración sobre investigaciones futuras. Es importante destacar que el Resumen no debe incluir ninguna información que no figure en el artículo original. ${ }^{3}$

Existen otros elementos que deben considerarse a la hora de preparar un Resumen 
para remitir a un evento científico; entre ellos se encuentran el título, autores, instituciones e iconografía.

Aunque el título debería ser el mismo del manuscrito completo, muchos eventos incluyen al título a la hora de la cuenta total de palabras / caracteres permitidos, por lo que puede ser necesario reducir su extensión, pero siempre manteniendo las características de un buen título: breve, específico, representativo e informativo.

La inclusión de autores en un Resumen debe responder a las consideraciones generales de autoría. ${ }^{4}$ Debe tenerse en cuenta que, al igual que con el título, muchos eventos incluyen los autores en la cuenta total de palabras / caracteres permitidos, lo cual se constituye en otro factor para valorar la verdadera pertinencia de la inclusión de cada autor. Igual consideración merece la descripción de la o las instituciones involucradas, un ítem en el cual es verdaderamente fácil ahorrar palabras/caracteres.

Aunque existen ocasiones en que se permite la inclusión de tablas o gráficos en el Resumen, tal circunstancia debe ser valorada muy cuidadosamente (y, de ser posible, desechada siempre). La inclusión de tablas/gráficos suele ser complicada, es fácil que se desordenen al momento de la remisión electrónica del Resumen, limitan el número de caracteres/palabras a incluir y su interpretación suele ser más complicada que lo que los autores sospechan.

Finalmente deben considerarse la inclusión de abreviaturas y referencias bibliográficas. Con respecto a las abreviaturas, su uso debe ser muy restringido y limitado a términos particularmente largos (que incluyan al menos tres palabras) y que se repitan frecuentemente (al menos tres veces); por supuesto, su explicación debe mencionarse en el cuerpo del Resumen la primera vez que aparece. En relación a las referencias bibliográficas, las mismas no tienen lugar en un Resumen. Una última consideración merecen los potenciales conflictos de interés: es importante mencionar el financiamiento recibido para la investigación.

Analizadas todas las consideraciones anteriores sobre lo que debe y no debe incluir el Resumen que se enviará a un evento científico, llegamos al punto práctico de cómo escribirlo. Una forma útil para comenzar es partir del trabajo completo e ir eliminando aquellas partes que no son imprescindibles para la comprensión del mismo. Partiendo de un manuscrito de 2000 palabras es fácil llegar así a un Resumen inicial de 500. A partir de allí se irá seleccionando los principales contenidos y, finalmente, se trabajará sobre la gramática a fin de lograr la comprensión de la idea fundamental con el menor número de palabras (200-300). Imaginemos que partimos de un bloque de mármol que queremos esculpir: inicialmente le daremos la forma general y luego iremos repasando los detalles una y otra vez hasta lograr el resultado deseado.

Aunque el destino principal del Resumen, tal cual lo hemos descripto, es la presentación de un trabajo en un evento científico, igual construcción merecen aquellos que encabezan un trabajo original remitido para su consideración a ser publicado en una revista científica, o los que preceden un proyecto remitido en búsqueda de financiamiento. ${ }^{5}$ A pesar de estar acompañados por el manuscrito completo con abundante información, en ambos casos el Resumen juega un papel fundamental. Es, sin lugar a dudas, lo primero que leerán los editores y árbitros de una revista, y los jurados de una convocatoria, y como tal les predispondrá el ánimo para continuar la lectura del material completo. Un Resumen bien escrito generará interés en quienes deben valorar un trabajo.

Independientemente de las consideraciones vertidas en este manuscrito, es importante revisar (y respetar) las instrucciones específicas que en cada caso existan para la preparación de cada Resumen que encaremos. También debe tenerse en cuenta que en muchas reuniones científicas se debe enviar el Resumen en inglés. Es importante revisar acabadamente la redacción en este idioma para incrementar la posibilidad de que el trabajo sea aceptado y también, de que sea adecuadamente comprendido. ${ }^{6}$

Finalmente, en esta época de comunicación abreviada debida a los recursos técnicos disponibles que ha generado una jerga de difícil comprensión, es importante verificar que lo que escribimos sea comprensible para todos aquellos a quienes queremos llegar.

\section{Dr. Fernando Ferrero Hospital General de Niños Pedro de Elizalde, Buenos Aires.}

http:/ /dx.doi.org/10.5546/aap.2015.104

\section{REFERENCIAS}

1. Pierson DJ. How to write an abstract that will be accepted for presentation at a national meeting. Respir Care 2004;49(10):120612.

2. Nakayama T, Hirai N, Yamazaki S, Naito M. Adoption of structured abstracts by general medical journals and format for a structured abstract. J Med Libr Assoc 2005;93(2): 237-42.

3. Day RA. Cómo preparar el resumen, en: Day R. Cómo escribir y publicar trabajos científicos. $3^{\text {a }}$ ed. en español. Washington DC: Organización Panamericana de la Salud; 2005. Capítulo 6, Cómo preparar el resumen; p.28-31.

4. Serra ME. Nuevas recomendaciones para sostener antiguos valores de responsabilidad y transparencia en publicaciones de revistas médicas. Arch Argent Pediatr 2013;111(6):466-7.

5. Subcomisión de Investigación. Guía para elaborar un proyecto de investigación. Arch Argent Pediatr 2011; 109(4):371-6.

6. Benfield JR, Feak CB. How authors can cope with the burden of English as an international language. Chest 2006;129(6):1728-30. 